\title{
Acoustic-speed correction of photoacoustic tomography by ultrasonic computed tomography based on optical excitation of elements of a full-ring transducer array
}

Jun Xia, Chao Huang, Konstantin Maslov, Mark A. Anastasio, Lihong V. Wang

Jun Xia, Chao Huang, Konstantin Maslov, Mark A. Anastasio, Lihong V. Wang, "Acoustic-speed correction of photoacoustic tomography by ultrasonic computed tomography based on optical excitation of elements of a full-ring transducer array," Proc. SPIE 8943, Photons Plus Ultrasound: Imaging and Sensing 2014, 89432G (3 March 2014); doi: 10.1117/12.2036615 


\title{
Acoustic-speed correction of photoacoustic tomography by ultrasonic computed tomography based on optical excitation of elements of a full- ring transducer array
}

\author{
Jun Xia, Chao Huang, Konstantin Maslov, Mark A. Anastasio, and Lihong V. Wang* \\ Department of Biomedical Engineering, Washington University in St. Louis, One Brookings Drive, \\ Saint Louis, Missouri 63130, USA \\ *Corresponding author: LHWANG@WUSTL.EDU
}

\begin{abstract}
Photoacoustic computed tomography (PACT) is a hybrid technique that combines optical excitation and ultrasonic detection to provide high resolution images in deep tissues. In the image reconstruction, a constant speed of sound (SOS) is normally assumed. This assumption, however, is often not strictly satisfied in deep tissue imaging, due to acoustic heterogeneities within the object and between the object and coupling medium. If these heterogeneities are not accounted for, they will cause distortions and artifacts in the reconstructed images. In this paper, we incorporated ultrasonic computed tomography (USCT), which measures the SOS distribution within the object, into our full-ring array PACT system. Without the need for ultrasonic transmitting electronics, USCT was performed using the same laser beam as for PACT measurement. By scanning the laser beam on the array surface, we can sequentially fire different elements. As a first demonstration of the system, we studied the effect of acoustic heterogeneities on photoacoustic vascular imaging. We verified that constant SOS is a reasonable approximation when the SOS variation is small. When the variation is large, distortion will be observed in the periphery of the object, especially in the tangential direction.
\end{abstract}

Keywords: Ultrasound computed tomography, photoacoustic computed tomography, image reconstruction, speed of sound

\section{INTRODUCTION}

Photoacoustic computed tomography (PACT) is a hybrid technique that is based on the acoustic detection of optical absorption in tissue from chromophores, such as oxygenated hemoglobin ( $\mathrm{HbO} 2)$ and deoxygenated hemoglobin $(\mathrm{Hb})$. The conversion of light to acoustic waves allows PACT to image vasculatures in deep tissues with acoustically defined high spatial resolution [1-8]. Over the past few years, several small-animal PACT systems have been developed with different detection geometries [1]. When small-animal whole-body vascular images were reconstructed in most cases, a uniform speed of sound (SOS) within the acoustic media was assumed. This assumption is violated in whole-body imaging due to the relatively large SOS variations within the animal body and between the coupling medium and the animal, which may cause distortions and artifacts in the reconstructed images.

To address this problem, one needs to measure the SOS variation of the medium, which can be achieved by ultrasonic computed tomography (USCT) $[9,10]$. However, most PACT systems lack transmitting electronics, partially due to the large number of dedicated receive preamplifiers connected right at the array output. While transmit-receive switches can be implemented, they add cost and complexity in electronic design, and also degrade the system's signal to noise ratio. Moreover, a transmitting ultrasonic array requires the usage of piezoelectric materials that are suboptimal for receiving photoacoustic signals. This problem was first addressed by S. Manohar et al., who suggested using the photoacoustic effect to generate ultrasonic waves from a point optical absorber [11]. In this study, we adapted a similar approach, but without using point absorbers. Taking advantage of a full-ring transducer array, we illuminated a narrow laser beam directly on the transducer surface. The laser beam is then scanned to provide sequential firing of array elements. With minimum modification, this method adds USCT capability to the PACT system.

Photons Plus Ultrasound: Imaging and Sensing 2014, edited by Alexander A. Oraevsky, Lihong V. Wang, Proc. of SPIE Vol. 8943, 89432G · C 2014 SPIE · CCC code: 1605-7422/14/\$18 · doi: 10.1117/12.2036615 


\section{SYSTEM DESIGN}

Figure 1(a) shows a schematic of the PACT system. The laser beam is first homogenized by a ground glass and then illuminated directly on the object in a 512-element full-ring transducer array. The array has a $5 \mathrm{MHz}$ central frequency, and the signals are detected by a data acquisition system with $40 \mathrm{MHz}$ sampling rate. A detailed description of the PACT system can be found in references [7, 12]. To perform USCT measurement, we developed a USCT add-on module, which consists of a conical lens and a round disk with a radial slit [Figure 1(b)]. The homogenized laser beam is first converted into a ring-shaped light and then passes through the $1 \mathrm{~mm}$ wide light slit to illuminate the transducer element. The conversion to ring-shaped light is essential for maximum light delivery efficiency and minimum light leakage to other elements. Compared with electronic-based ultrasound firing, our approach has two advantages. First ultra-short laser pulses can generate clean, high intensity broad-band ultrasonic signals, which are favorable in frequency-domain data analysis, such as attenuation measurement. Second, USCT can be performed using the same laser for PACT experiments, simplifying system design. By rotating the light slit, we could sequentially fire different array elements to perform USCT measurement. In this study, we used only one light slit. However, multiple light slits can be applied to speed up measurement, as long as signals from different firing elements can be separated in time.
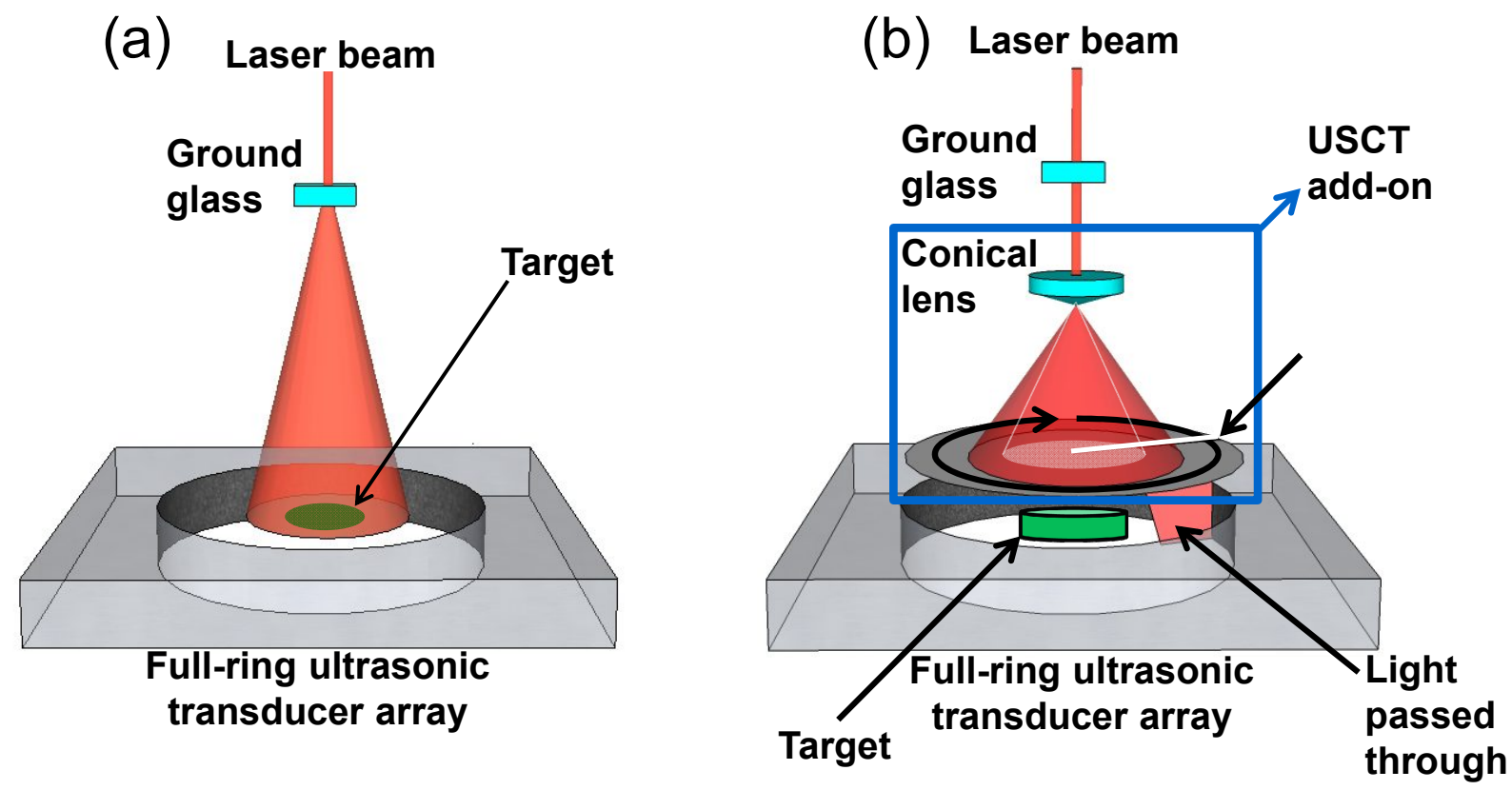

Figure 1. (a) Schematic of the photoacoustic computed tomography system. (b) Schematic of the integrated photoacoustic and ultrasonic computed tomography system.

\section{RESULTS}

We first used numerical simulations to demonstrate the distortion of images due to acoustic heterogeneities. The simulation was performed using the k-Wave toolbox [13]. To mimic vascular structures, we used a skeleton leaf [Figure 2a], embedded in a circular gel with sound speeds higher than that of water. A skeleton leaf was used because it has rich vascular structures and the simulation can be validated experimentally. We simulated four cases, with two different sound speeds [Figures 2(b) and 2(c)] and two different gel diameters (Figure 2, top and bottom rows). The scanning geometry is similar to that in our experimental setup: 512 transducer elements evenly distributed in a 5-cm-diameter ring. The photoacoustic images were reconstructed using a constant SOS (the optimum SOS), derived by maximizing the image sharpness function [13]. This is also the optimization procedure we used when imaging an unknown object in experimental settings. From Figures 2(b) and 2(c), we can see that the distortion is negligible in the small object 
(diameter: $10 \mathrm{~mm}$ ). When the diameter of the object increases to $20 \mathrm{~mm}$ (a typical size of a mouse cross section), we start to see blurring in the periphery of the object. It is also worth noticing that the level of blurring is different for vessels along different directions: a vessel along the radial direction blurs more than a vessel along the tangential direction. Detailed explanation of this phenomenon can be find in reference [9].

(a)

\section{Numerical phantoms}
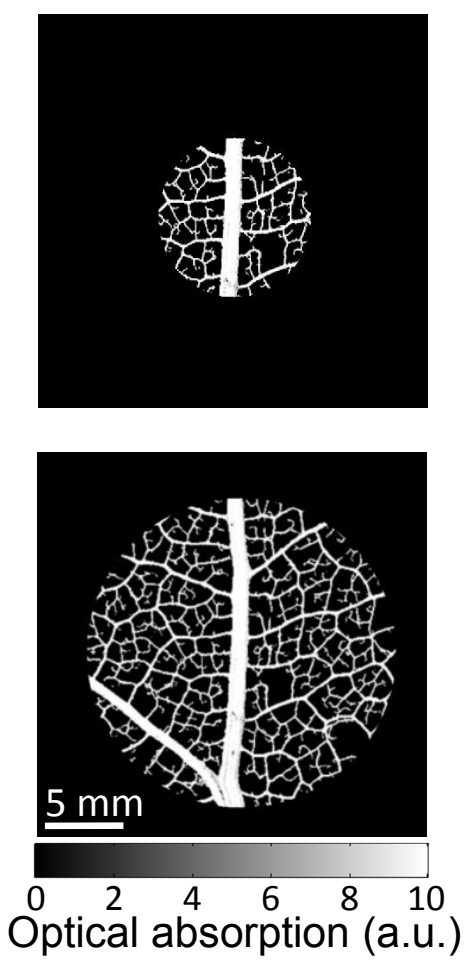

(b)
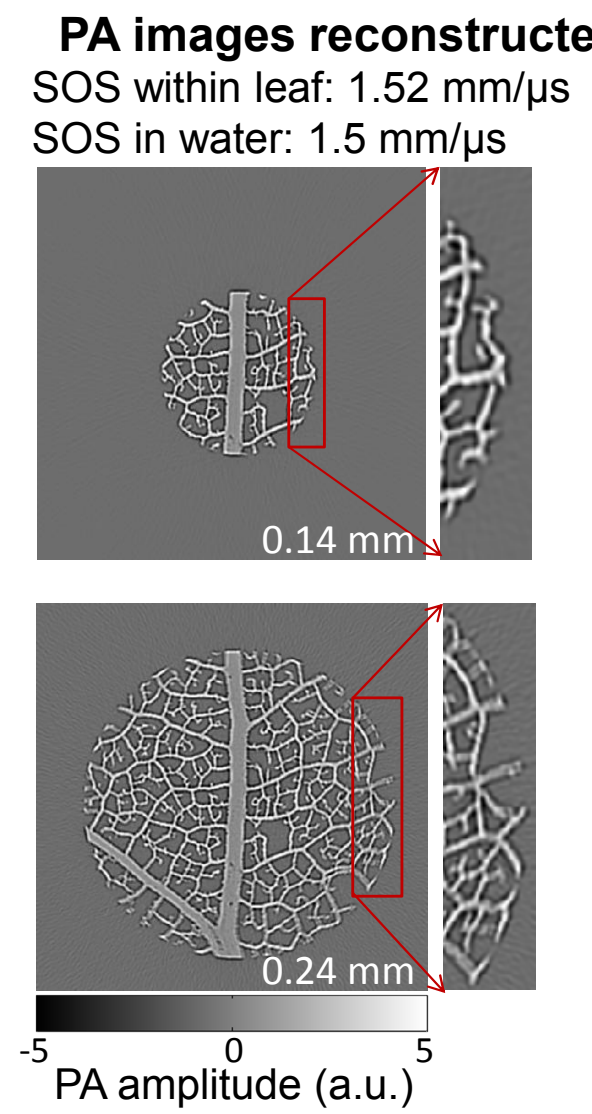

(c)
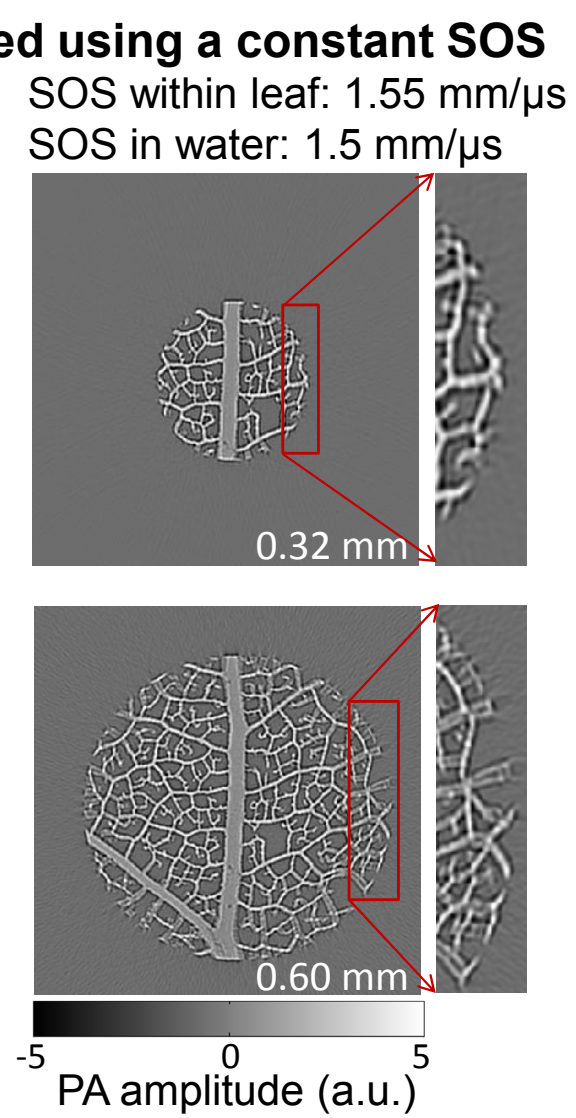

Figure 2. Numerical phantom studies. (a) Numerical leaf phantoms showing the distribution of optical absorption in arbitrary units. (b) and (c), photoacoustic images reconstructed using a constant speed of sound that renders the sharpest image. The initial pressure data was simulated by assigning the speed of sound within the leaf to be $1.52 \mathrm{~mm} / \mu \mathrm{s}$ and 1.55 $\mathrm{mm} / \mu \mathrm{s}$ in (b) and (c), respectively. The speed of sound in water is $1.5 \mathrm{~mm} / \mu \mathrm{s}$ for all cases. The number written at the bottom right corner of each image is the observed vessel split.

We then investigated the effect of acoustic heterogeneity using the integrated USCT-PACT system. The first object was a mouse brain embedded in an agar gel. We initially performed the PACT experiment [Figure 3(a)] and then added the USCT module to get the SOS map [Figure 3(b)]. The mouse brain and the cylindrical agar gel can be clearly identified in the SOS map. This SOS map was then input to the k-Wave toolbox for time-reversal PACT image reconstruction [Figure 3(c)]. Compared with the PACT image reconstructed using the optimum SOS [Figure 3(a)], the SOS-informed reconstruction [Figure 3(c)] looks very similar. This is mainly because the periphery of the brain does not contain many vessels along the radial direction. The composite image [Figure 3(d)] indicates that the system can provide multiple contrasts. 

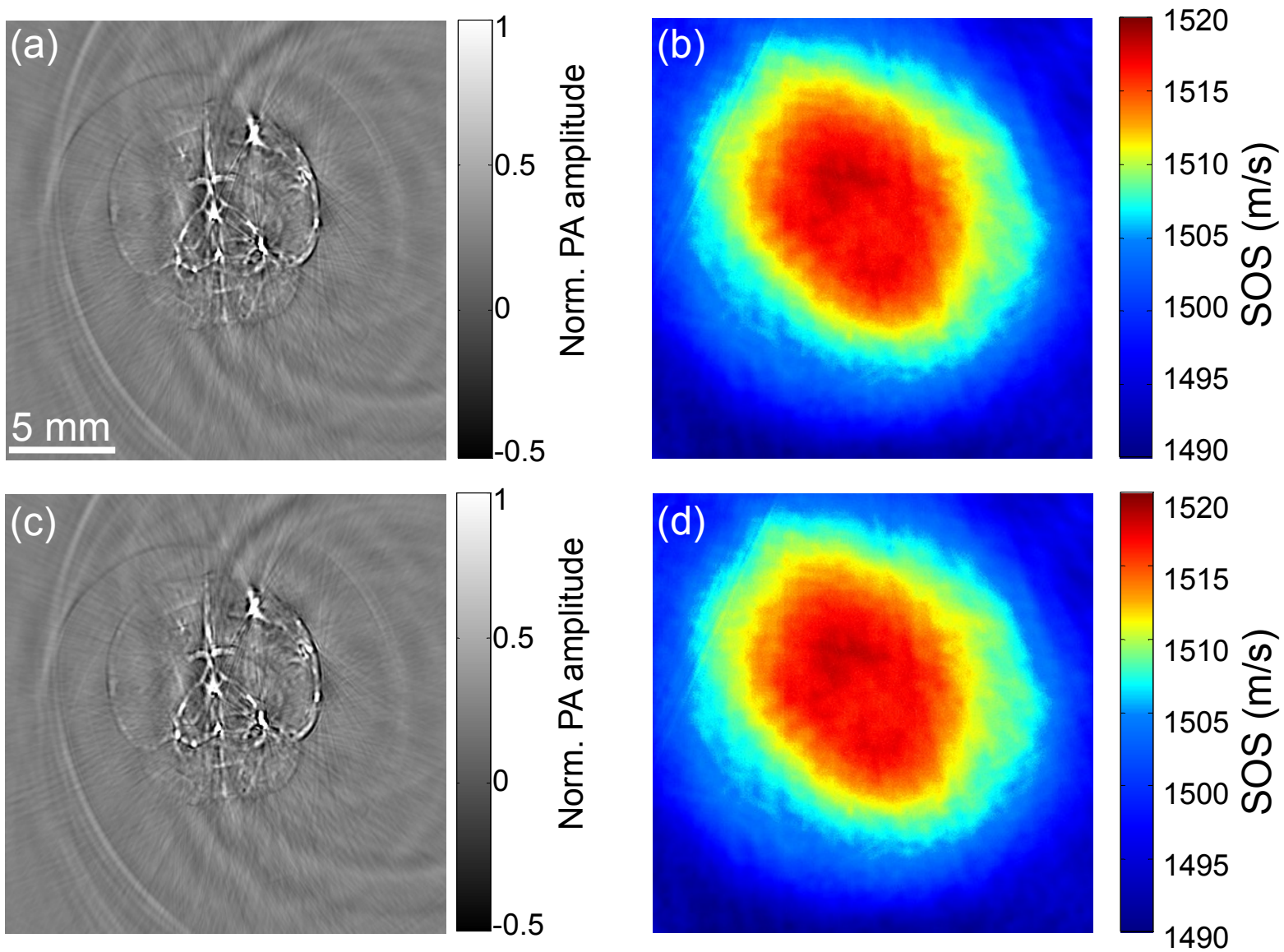

Figure 3. Experimental images of an excised mouse brain. (a) PACT image reconstructed using a constant SOS that renders the sharpest image. (b) The SOS map obtained from USCT measurement. (c) PACT image reconstructed using the SOS map in (b). (d) Composite image with the PACT image in (c) overlaid on the SOS map.

We further studied the acoustic heterogeneity effect using a skeleton leaf phantom [Figure 4(a)]. To increase the SOS in the agar gel, we mixed the agar solution with $1 \%$ glycerol (SOS: $1.90 \mathrm{~mm} / \mu \mathrm{s}$ ), yielding an agar gel with an SOS around $1.51 \mathrm{~mm} / \mu \mathrm{s}$. We performed PACT and USCT experiments in sequence as in the previous brain phantom study. The images were first reconstructed using the optimum SOS [Figures 4(b)]. Because of the small SOS difference, vessel splitting is not significant. We then performed time-reversal reconstruction using the SOS map [Figure 4(c)]. As expected, the image [Figures 4(d)] looks similar to Figure 4(b). The study indicates that when the SOS different is small, constant SOS is a reasonable approximation. In reference [9], the leaf was embedded in a agar gel with even higher SOS. In that case, vessel splitting along the tangential direction can be clearly observed. 
(a)

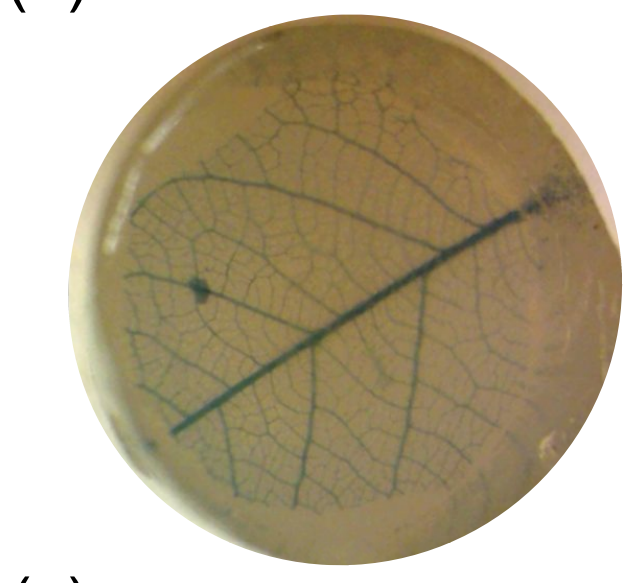

(b)

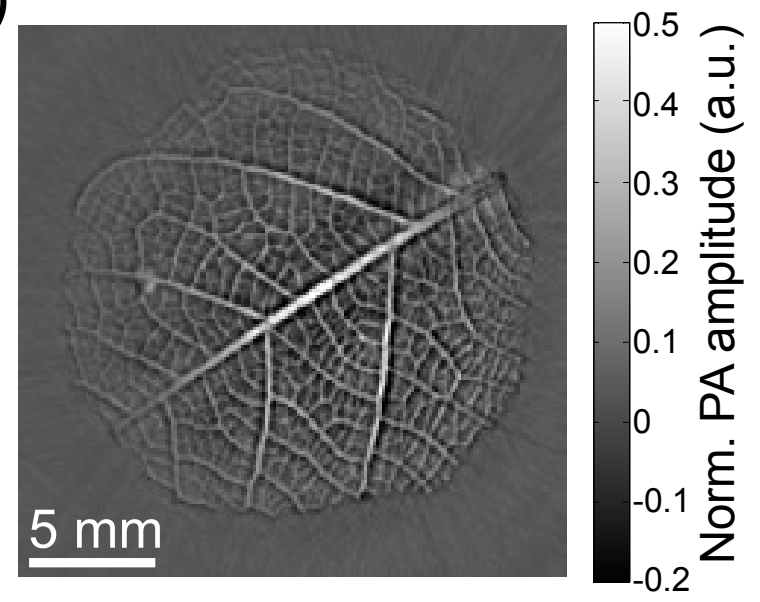

(c)

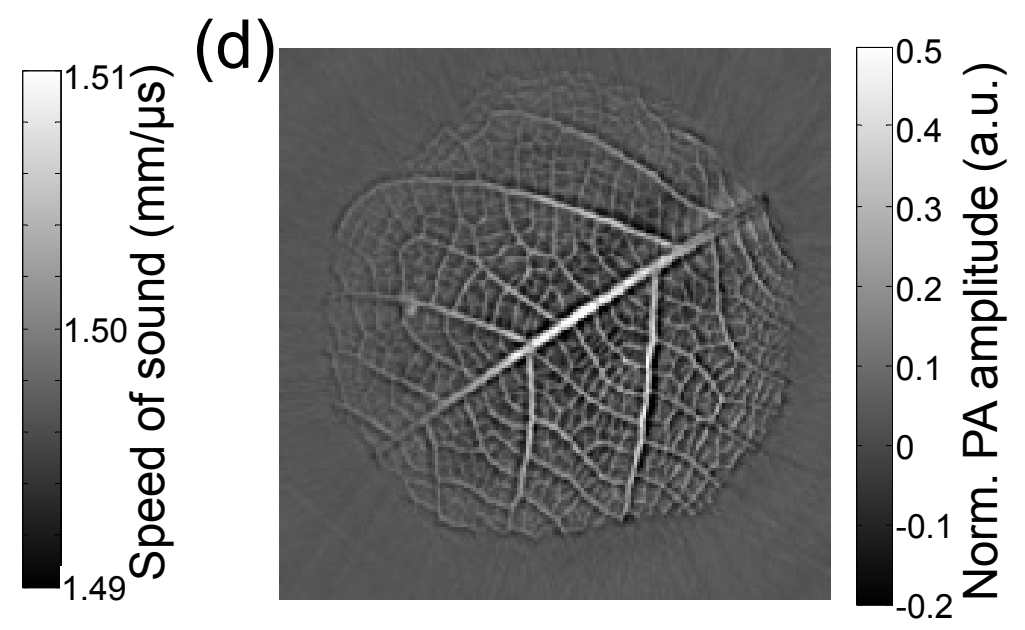

Figure 4. Experimental results of a leaf phantom. (a) Photograph of the skeleton leaf embedded in an agar gel with SOS of $1.535 \mathrm{~mm} / \mu \mathrm{s}$. (b) PACT image reconstructed using a constant average speed of sound (SOS), which was optimized for image sharpness. (c) The SOS map obtained from USCT measurement. (d) PACT image reconstructed using the SOS map in $(\mathrm{d})$.

\section{SUMMARY}

We developed an integrated USCT and PACT system based on a full-ring ultrasonic transducer array. USCT firing was performed using the same laser as for PACT experiments. The ultra-short laser pulse allows generating clean broad-band acoustic waves. The system was used to study the distortion of PACT images due to acoustic heterogeneity between the object and the coupling medium. We find that constant SOS is a reasonable approximation when the SOS variation is small or when the object is small compared to the scanning geometry. For larger objects and larger SOS variations, the image reconstructed using constant SOS will be distorted, mainly at the periphery of the object and along the tangential direction. An integrated USCT and PACT system substantially improves the accuracy of PACT imaging reconstruction. 


\section{REFERENCES}

[1] J. Xia, and L. Wang, "Small-animal whole-body photoacoustic tomography: a review," Biomedical Engineering, IEEE Transactions on, PP(99), 1-1 (2013).

[2] J. Xia, G. Li, L. Wang et al., "Wide-field two-dimensional multifocal optical-resolution photoacousticcomputed microscopy," Optics Letters, 38(24), 5236-5239 (2013).

[3] J. Xia, A. Danielli, Y. Liu et al., "Calibration-free quantification of absolute oxygen saturation based on the dynamics of photoacoustic signals,” Opt. Lett., 38(15), 2800-2803 (2013).

[4] M. Nasiriavanaki, J. Xia, H. Wan et al., "High-resolution photoacoustic tomography of resting-state functional connectivity in the mouse brain," Proc Natl Acad Sci U S A, 111(1), 21-6 (2014).

[5] J. Xia, W. Chen, K. Maslov et al., "Retrospective respiration-gated whole-body photoacoustic computed tomography of mice," Journal of Biomedical Optics, 19(1), 016003-016003 (2014).

[6] M. R. Chatni, J. Xia, R. Sohn et al., "Tumor glucose metabolism imaged in vivo in small animals with wholebody photoacoustic computed tomography," Journal of Biomedical Optics, 17(7), 076012 (2012).

[7] J. Xia, Z. Guo, K. Maslov et al., "Three-dimensional photoacoustic tomography based on the focal-line concept," Journal of Biomedical Optics, 16(9), 090505 (2011).

[8] J. Yao, J. Xia, K. I. Maslov et al., "Noninvasive photoacoustic computed tomography of mouse brain metabolism in vivo," NeuroImage, 64, 257-266 (2013).

[9] J. Xia, C. Huang, K. Maslov et al., "Enhancement of photoacoustic tomography by ultrasonic computed tomography based on optical excitation of elements of a full-ring transducer array," Opt. Lett., 38(16), 3140-3143 (2013).

[10] J. Jose, R. G. H. Willemink, W. Steenbergen et al., "Speed-of-sound compensated photoacoustic tomography for accurate imaging," Medical Physics, 39(12), 7262-7271 (2012).

[11] J. Jose, R. G. H. Willemink, S. Resink et al., "Passive element enriched photoacoustic computed tomography (PER PACT) for simultaneous imaging of acoustic propagation properties and light absorption," Opt. Express, 19(3), 2093-2104 (2011).

[12] J. Xia, M. Chatni, K. Maslov et al., "Whole-body ring-shaped confocal photoacoustic computed tomography of small animals in vivo," Journal of Biomedical Optics, 17(5), 050506 (2012).

[13] B. E. Treeby, and B. T. Cox, "k-Wave: MATLAB toolbox for the simulation and reconstruction of photoacoustic wave fields," Journal of Biomedical Optics, 15(2), (2010). 